\title{
Estudio de la significación de la inseguridad ambiental en dos casos en Córdoba, Argentina
}

\author{
Study of the significance of environmental insecurity in two \\ cases in Córdoba, Argentina
}

\section{Estudo da significação da insegurança ambiental em dois casos em Córdoba, Argentina}

Erika Saccucci

Profesora adjunta en la cátedra de Epistemología de las Ciencias Sociales, Universidad Católica de Córdoba, Becaria posdoctoral CONICET-Área de Ciencias Sociales y Humanidades, Universidad Católica de Córdoba, Córdoba, Argentina. Correo: erika_sac34@hotmail.com.

doi:10.18472/SustDeb.v10n1.2019.19137

\section{RESUMEN}

En este artículo se ha realizado un estudio de la significación de la polución en dos conflictos articulados en torno a la cuestión ambiental: Pueblos Unidos y VUDAS- Vecinos Unidos en Defensa de un Ambiente Seguro de Córdoba, Argentina. El sufrimiento ambiental no es experimentado en aislamiento, sino dentro de contextos discursivos y en relaciones de poder. Estos contextos dan forma a los caminos en los cuales la aflicción es vivida y comprendida en cada caso. A partir del análisis de contenido de veinte entrevistas en profundidad concluimos que en los conflictos analizados se construyen dos sentidos opuestos en relación a la polución: mientras que en Pueblos Unidos el peligro de enfermedades por polución es relativizado, en un contexto de ausencia de otras oportunidades para garantizar la vida, en VUDAS, la polución es la estructurante principal de la lucha ya que denuncian su presencia y la enfermedad de los cuerpos.

Palabras clave: Conflictos; Polución; Precariedad; Inseguridad Ambiental; Análisis de contenido.

\section{RESUMO}

Vizinhos Unidos em defesa de um ambiente seguro em Córdoba, Argentina. O sofrimento ambiental não é experimentado isoladamente, mas dentro de contextos discursivos e de relações de poder. Esses contextos dão forma às maneiras pelas quais a aflição é vivida e compreendida em cada caso. Com base na análise de conteúdo de vinte entrevistas em profundidade, concluímos que, nos conflitos analisados, são construídos dois sentidos opostos em relação à poluição: enquanto em Pueblos Unidos, o perigo de doenças como efeto da poluição é relativizado, num contexto de ausência de outras oportunidades para garantir a vida, em VUDAS, a poluição é a principal estruturante da luta ao denunciar a sua presença e a doença dos corpos.

Palavras-Chave: Conflitos; Poluição; Precariedade; Insegurança Ambiental; Análise de Conteúdo. 


\section{INTRODUCCIÓN}

El desarrollo de la sociología medio ambiental en el mundo emerge en los años 60 como una subdisciplina de creciente importancia en la sociología académica. Los años 60 representan un momento de crisis que expresan tensiones con el paradigma de la modernidad, la idea del progreso y el desencanto de la idea de superación de las desigualdades sociales a través de un proceso de tecnificación y avance del conocimiento científico. Entre la diversidad de movimientos y denuncias que cobraron relevancia en esta década, las críticas realizadas al modo de acumulación capitalista y su vinculación con la polución hacen que se hable de los sesenta como la década del medio ambiente.

En el proceso de desarrollo de la investigación sociológica sobre las cuestiones medioambientales, se han analizado diversos aspectos de la relación entre la sociedad y el medio ambiente, así como también se registran diversas perspectivas empleadas (OLTRA ALGADO, 2006). Los valores sobre el medio ambiente, la aparición del movimiento ecologista, la economía política de la degradación medioambiental, las políticas del medio ambiente o el riesgo tecnológico han sido algunos de los grandes temas tratados por la sociología medioambiental hasta la fecha (BUTTEL, 1987).

La cuestión medioambiental ha estimulado la investigación empírica así como también la elaboración teórica, en muchos casos realizada desde ámbitos no estrictamente de la sociología medioambiental y con gran influencia en toda la sociología (GIDDENS, 1990; BECK, 1998). Así, la temática medioambiental recibió gran atención en los sesenta, se distendió en los 70 y 80, para adquirir nuevamente auge en los noventa (DUNLAP, 2002).

A los fines de esta investigación interesan específicamente los estudios que abordan la polución y la vinculación que diversas comunidades establecen con ella. Existe una dimensión social y cultural de los problemas ambientales (DOUGLAS Y WILDAVSKY, 1982), que posee el mismo estatuto de realidad que la derivada de su dimensión física. Esta forma de existencia de los problemas depende de un proceso social de construcción y tiene que ver con su percepción, significación y reconocimiento público. Quienes han reflexionado acerca de los problemas ambientales, desde el punto de vista de las ciencias sociales, tratan de explicar los mecanismos por medio de los cuales determinados aspectos de la realidad adquieren significado colectivo especial, que los hace surgir en el escenario público (LEZAMA, 2001).

En relación a la construcción cultural y social de las problemáticas medioambientales es posible reconocer diversas perspectivas, que se enuncian a continuación. Es preciso aclarar que no se pretende una descripción de todas las perspectivas que han abordado la problemática medio ambiental, lo cual excedería el objetivo de este trabajo, sino más bien, recuperar solo aquellas que puedan entrar en discusión directa con la perspectiva aquí presentada. Así, por una parte, encontramos aquella que sostiene que las sociedades seleccionan los tipos de problemas de los cuales desean preocuparse y aquellos que deciden ignorar. Este proceso selectivo se produce al mismo tiempo cuando se eligen las instituciones, los valores y las normas en las que una sociedad decide vivir. Esta perspectiva racionalista supone que los sujetos deciden "libremente", con plena información, qué temáticas son relevantes y preocupantes y cuáles no lo son.

En segundo lugar hemos encontrado la corriente teórica llamada posmaterialista que aduce que, las sociedades solo comienzan a preocuparse de temas como las condiciones medio ambientales cuando cuentan con sus necesidades materiales y fundamentales cubiertas. En otras palabras, una vez satisfechas las necesidades más elementales, las sociedades comienzan a preocuparse un poco más por demandas cualitativas, como son las que tienen que ver con el medio ambiente (INGLEHART, 1971). Desde esta perspectiva, solo los países considerados "del primer mundo" presentan esta preocupación.

Desde la perspectiva propuesta a los fines de esta investigación hemos sostenido que hay una separación entre daño y conciencia que está definida por su mediación simbólica y social. Sin embargo, no se considera que la mediación simbólica y social refiera a un proceso absolutamente racional de aquello considerado relevante o contaminante. Del mismo modo, la idea de que la preocupación por lo medio 
ambiental cobra relevancia en aquellas sociedades que cuentan con una base material garantizada no permite explicar los movimientos medio ambientales que se han desarrollado con gran fuerza en toda América Latina. Por el contrario, aquí se ha propuesto que cada comunidad significa de modo diverso su relación con el medio ambiente y la polución.

El estudio de las condiciones medio ambientales y el modo en el cual las comunidades significan el sufrimiento ambiental es una preocupación marginal entre los estudiosos de la pobreza en América Latina. Solo se registra esta problemática en algunos trabajos (LEMOS 1998; PEZZOLI 2000; EVANS 2002; HOCHSTETLER Y KECK 2007; AUYERO Y SWISTUN, 2008; BERGER, 2013). Estos buscan comprender los modos y contextos en que los actores sociales involucrados en conflictos socioambientales construyen la noción de "riesgo medioambiental" y qué actuaciones asumen en él.

De acuerdo a Auyero y Swistun (2008) el sufrimiento hace sentido en tanto experiencia individual, ya que el padecimiento es parte de lo vivenciado por el cuerpo, pero a la vez es social ya que las situaciones de sufrimiento son construcciones colectivas ancladas en contextos relacionales y discursivos que moldean culturalmente las formas en las que los actores viven y entienden su dolor y las causas que lo producen. El sufrimiento ambiental debe ser entendido como una forma particular de sufrimiento social causado por las acciones contaminantes concretas de actores específicos. En otras palabras, el sufrimiento, en este caso el ambiental, no es experimentado en aislamiento, sino dentro de contextos discursivos y en relaciones de poder. Estos contextos dan forma a los caminos en los cuales la aflicción es vivida y comprendida.

Los sujetos no piensan y sienten lo tóxico de una manera única y monolítica: se presenta una diversidad de visiones y creencias que coexisten (a veces en el mismo individuo). No hay ni una población determinada a hacer algo en contra de la agresión tóxica, ni una población completamente acostumbrada a la polución (AUYERO Y SWISTUN, 2007).

Desde esta perspectiva se ha propuesto en este artículo el estudio de la significación de la polución en dos conflictos articulados en torno a la cuestión ambiental: Pueblos Unidos y VUDAS- Vecinos Unidos en Defensa de un Ambiente Seguro. En los conflictos analizados se construyen dos sentidos opuestos en relación a la polución: mientras que para los primeros el peligro de enfermedades por polución es relativizado, en un contexto de ausencia de otras oportunidades para garantizar la vida, en el segundo, la polución es la estructurante principal de la lucha ya que denuncian su presencia y la consiguiente enfermedad de los cuerpos.

En el apartado siguiente se exponen las principales decisiones metodológicas, para luego presentar el análisis de las entrevistas en profundidad, donde emergen las dos significaciones sobre la polución ya señaladas.

\section{LOS CASOS ESTUDIADOS Y LAS DECISIONES METODOLÓGICAS}

A los fines de esta investigación se han seleccionado dos conflictos en donde se presentan discusiones vinculadas a las condiciones medioambientales: Pueblos Unidos y VUDAS, en contra de la permanencia de la empresa Porta Hermanos (hnos).

Pueblos Unidos es una toma de tierra que data del año 2007 cuando diversas familias se organizaron a los fines de avanzar sobre tierras disponibles que habían sido un basural a cielo abierto en los años 70 y aún presentaban grandes cantidades de residuos.

La mayoría de las familias que realizaron esta toma de tierra son de origen extranjero, principalmente Perú y Bolivia y una minoría de Paraguay y Argentina que, por lo general, ya vivían en la ciudad pero provenían de otras zonas. En su mayoría trabajadores de la construcción, comerciantes, empleadas domésticas y trabajadoras textiles a encargo. En la actualidad en el barrio vive un número aproximado de 700 familias (MACHADO IBARS, 2017).

De esta manera, las y los vecinos se organizaron de manera autogestiva a los fines de garantizar la limpieza de la tierra. Esta consistió en la remoción de grandes cantidades de basura y renovación de 
la tierra. La toma de tierra se desarrolló sin mayores dificultades y las y los vecinos avanzaron sobre la construcción en material de sus viviendas, en el trazado de calles de acuerdo a las disposiciones urbanas y en la lucha por los servicios. En 2009 se inició un proceso conflictivo a partir de la intervención de diversos actores que sostenían que la tierra se encontraba contaminada.

Así, la Universidad Nacional de Córdoba (Cátedra de Ingeniería Ambiental de la Facultad de Ciencias Exactas, Físicas y Naturales) en articulación con la Universidad Tecnológica Nacional UTN, realizó un estudio en donde detectó altos niveles de polución con metales pesados, plomo y cromo. Además, recomendaba que se realizara un proceso de remediación ambiental si lo que se pretendía era un uso residencial del terreno (LA VOZ DEL INTERIOR, 2017).

Para la realización del estudio, la comunidad barrial acordó estar presente en el momento de toma de las muestras de tierra, a los fines de verificar su origen y asegurarse que las muestras fuesen tomadas de diversos puntos del barrio. Sin embargo, este acuerdo no fue respetado y las y los vecinos no pudieron participar del proceso. Por esta razón, una parte del barrio impugna los resultados del estudio ya que consideraron poco transparente el procedimiento y descreen que la tierra analizada haya sido efectivamente tomada en el barrio.

Por otra parte, la comisión de Salud Pública, en articulación con el dispensario de Nuestro Hogar III (UPAS 15), tomó 40 muestras de sangre de niños de la comunidad. Luego de su análisis anunció que el $35,7 \%$ de las muestras presentaba plomo en sangre (LA VOZ DEL INTERIOR, 2009). Este estudio fue repudiado por la comunidad barrial ya que de las 40 muestras solo anunciaron resultados de 28 y no se proveyó a las familias de un resultado formal, por escrito, sino que su anuncio fue oral.

El municipio de la ciudad de Córdoba, basado en estos estudios, presentó ante el Concejo Deliberante un proyecto de expropiación de un terreno próximo, ubicado en Camino San Antonio, con el objetivo de trasladar las familias. Frente a esto, la mayoría de las y los vecinos se opusieron ya que sostenían que la gran cercanía de los terrenos entre sí y el hecho de que estos hayan sido usados para la producción de soja y, por ende fumigados, hacía poco probable que se encontrasen libres de polución.

De esta manera, la comunidad barrial puede ser dividida entre quienes aceptan la idea de la presencia de polución pero carecen de otras opciones y quienes niegan la veracidad de estos estudios y sospechan que se busca "liberar" la tierra a los fines de construir un emprendimiento inmobiliario, o bien, destinarlo nuevamente para el enterramiento de basura y así resolver de manera permanente la problemática estructural de los desechos de la basura en la ciudad.

Finalmente, en diciembre de 2010 el Concejo Deliberante de la ciudad declaró a Nuestro Hogar IV como "inhabitable" a través de una ordenanza (ORDENANZA N.o 3156-E-10). Esta declaración resulta de fundamental importancia en tanto representa el primer caso en el país. Aquello que distingue este conflicto es la discusión en torno a "lo habitable" y al conocimiento científico.

A diferencia de aquellos conflictos que se organizan en torno a la defensa del ambiente y que pueden ser denominados como conflictos ambientales, este caso se erige por la defensa de la tierra conseguida a través de su posesión y pone en cuestión la idea de polución y las condiciones que hacen posible la vida.

Por su parte, el conflicto de VUDAS- Porta hnos. afecta a los vecinos de los barrios Inaudi, San Antonio, Posta de Vargas, Cañitas y 2 de Abril. Porta Hermanos instaló la planta de producción de alcohol en el año 1995, unas cuatro décadas después de la formación del barrio Parque San Antonio. Sin embargo, en ese momento la regulación de suelo la definía como apta para actividades industriales. Posteriormente la categorización cambió y desde entonces la planta recibe extensiones en sus permisos de funcionamiento (LA VOZ DEL INTERIOR, 2014).

El conflicto se inició en 2012 cuando las y los vecinos escucharon sonidos de explosión provenientes de la fábrica. Allí se enteraron que la empresa Porta Hnos. que hasta el momento producía alcohol y sus derivados, había comenzado a producir bioetanol. Con el pasar del tiempo los olores nauseabundos y la aparición de múltiples afecciones como problemas respiratorios, alergias y 
problemas en la piel atrajeron la atención de las vecinas que comenzaron a informarse sobre las consecuencias de este tipo de producción.

La recurrencia de las enfermedades y la aparición de casos de cáncer, púrpura y abortos espontáneos las llevó a contactarse con REDUAS (Red Universitaria de Ambiente y Salud) quienes realizaron un primer relevamiento de las afecciones de la zona. La encuesta detectó que 43,2 por ciento de los vecinos tenía cefaleas persistentes y 34,8 por ciento sufría de conjuntivitis y congestión ocular, por encima de la media parámetro (CBA 24N, 2013). A su vez, la articulación con Madres de Barrio Ituzaingó1 resultó clave para que las vecinas organizadas vinculasen la actividad económica con la presencia de las afecciones. El "mapa de la muerte" elaborado por las Madres de Ituzaingó vincula el uso de químicos a la aparición de las enfermedades y puede hacerse extensivo a la experiencia de VUDAS. De esta manera, las vecinas organizadas en VUDAS reclaman la relocalización de la planta hacia zonas no habitadas.

En 2013 los vecinos realizaron una denuncia penal contra la empresa por polución, la cual quedó a cargo del fiscal José Mana que ordenó una pericia científica (ECOS CÓRDOBA, 2013). Esto resultó de relevancia en tanto era la primera vez que en el fuero penal se realizaba una pericia para medir la polución en el aire. La pericia oficial se realizó por el Centro de Vinculación de Tecnología Química Industrial (Cetequi), de la Facultad de Ciencias Exactas Físicas y Naturales de la Universidad Nacional de Córdoba a cargo del Ingeniero Yorio quien tiene una cátedra de extensión en articulación con la empresa (ECOS CÓRDOBA, 2015). Finalmente, los resultados fueron inconcluyentes ya que "no había pruebas suficientes que pudiesen vincular las afecciones con la empresa" por lo cual, la causa fue archivada.

A partir del desarrollo del conflicto, el discurso científico ocupa una centralidad fundamental. Así, La característica principal de este conflicto es la disputa en torno a los resultados de los estudios que han implicado una discusión dentro de VUDAS en torno a las formas de medición y a los vínculos entre el conocimiento y el poder.

En el 2016 las vecinas presentaron un amparo ambiental contra la empresa Porta y los Ministerios de Ambiente y Energía de la Nación, el cual fue rechazado en septiembre del 2017 (LA TINTA, 2017). Ante la apelación de los vecinos, la Cámara Federal ordenó reabrir el caso. Resulta necesario destacar que se trata del segundo caso de amparo ambiental en todo el país.

La selección de estos casos se realizó sobre la base de una compilación de los diversos conflictos articulados en torno al ambiente en Córdoba. Se ponderó la diversidad de los casos y que presentaran elementos novedosos para los estudios medio-ambientales. Así, el caso de Pueblos Unidos o Nuestro Hogar IV se distingue por la singular discusión en torno a las condiciones medioambientales en relación a la protección de la vida. A los fines de este artículo nos hemos centrado en aquellos discursos que aceptan la presencia de polución pero relativizan sus efectos sobre el cuerpo en un contexto de escasez total de otras oportunidades. Por su parte, el conflicto VUDAS- Porta refiere al único caso en el mundo en el que se registra la presencia de una planta productora de Bioetanol dentro del éjido urbano, directamente colindante con casas de uso residencial y de gran densidad poblacional.

Resulta necesaria realizar una distinción conceptual entre contaminación y polución. Mientras que la contaminación refiere a la presencia de agentes físicos o químicos que afectan nocivamente un medio concreto, la polución refiere a la presencia de agentes o actividades que producen un daño significativo en el ambiente y dañan drásticamente el ecosistema. En otras palabras, la polución es la introducción en el ambiente de una substancia o elemento que tiene efectos peligrosos o tóxicos. Así, toda polución es contaminación, pero no toda contaminación es polución".

Para la realización de esta investigación hemos elegido el "estudio de casos múltiples" como estrategia de análisis, donde los casos fueron intencionalmente seleccionados en relación a su importancia estratégica, tanto en términos teóricos como empíricos.

De acuerdo con Muñiz (2010) existen diversas perspectivas en torno al uso de los estudios de caso. Para algunos investigadores se vincula con un enfoque de investigación, el "caso" constituye el organizador de las diferentes dimensiones de análisis que informa y facilita la construcción de teorías. A su vez, los estudios de caso pueden concebirse como parte de la técnica de recolección de información, el modo 
de selección de la muestra. Por último, y como aquí proponemos, estos pueden ser abordados como una "estrategia de investigación cualitativa" (VASILACHIS DE GIARDINO, 2005).

El estudio de casos múltiples consiste en la selección de unidades de análisis orientada tanto por la relevancia empírica que estas presentan, así como también por dimensiones teóricas que requieren ser informadas por los datos. Esta selección -intencional- consiste en incorporar aquellos casos que ofrezcan una serie de particularidades en su interior que ponen en tensión, ensanchan y crean nuevas interpretaciones o marcos conceptuales. Como señalan Neiman y Quaranta (2006, p. 216):

A diferencia de la grounded theory, [el estudio de caso] puede partir de la utilización de categorías conceptuales para el desarrollo de la investigación y el desarrollo de teoría y, a diferencia de la etnografía, realiza recortes específicos de la realidad social para su abordaje; a la vez que no se establecen, necesariamente, instancias de reflexividad formalmente formuladas con respecto a la participación del investigador en terreno (NEIMAN y QUARANTA, 2006, p. 216).

Para el estudio de estos conflictos se han realizado 20 entrevistas en profundidad a través de la implementación de la técnica de la bola de nieve. La cantidad de entrevistas realizadas varía en cada caso estudiado. Dicha variación responde al criterio de saturación teórica donde los datos que emergen de las entrevistas se hacen repetitivos, o similares (ÁLVAREZ GAYOU, 2003).

A su vez, para la mejor comprensión de cada uno de los casos, y su historización, apelamos a registros de campo elaborados a partir de conversaciones con las y los vecinos, o de la participación en eventos o actividades por ellos convocados (conferencias, charlas, marchas, intervenciones públicas), así como también, se realizó una recolección documental que incluye notas periodísticas sobre los conflictos y materiales de difusión producidos por las organizaciones en lucha: folletería, cartas y material en redes sociales. A continuación se presenta una tabla resumen con información sobre las y los entrevistados:

Tabla 1 - Información sobre las y los entrevistados.

\begin{tabular}{|c|c|c|c|c|c|c|}
\hline Número & Fecha & Lugar & $\begin{array}{l}\text { Duración de } \\
\text { la entrevista }\end{array}$ & Datos & Código & Caso \\
\hline 1 & $22 / 12 / 2014$ & $\begin{array}{l}\text { Casa de dos de los } \\
\text { entrevistados }\end{array}$ & $46^{\prime}$ & $\begin{array}{l}\text { Los tres son peruanos } \\
\text { (dos hombres y una } \\
\text { mujer). Viven en una } \\
\text { casa construida por la } \\
\text { ONG un Techo para mi } \\
\text { País }\end{array}$ & $\begin{array}{l}\text { TOMA - V01 - } \\
\text { Pueblos Unidos }\end{array}$ & $\begin{array}{l}\text { Pueblos } \\
\text { Unidos }\end{array}$ \\
\hline 2 & 05/09/2014 & $\begin{array}{l}\text { Casa de la } \\
\text { entrevistada }\end{array}$ & $40^{\prime}$ & $\begin{array}{c}\text { Mujer peruana, vive en } \\
\text { la manzana "más vieja" } \\
\text { de la toma, hace aprox. } \\
1 \text { año }\end{array}$ & $\begin{array}{l}\text { TOMA - V02 - } \\
\text { Pueblos Unidos }\end{array}$ & $\begin{array}{l}\text { Pueblos } \\
\text { Unidos }\end{array}$ \\
\hline 3 & 05/09/2014 & $\begin{array}{l}\text { Casa de la } \\
\text { entrevistada }\end{array}$ & $59^{\prime}$ & $\begin{array}{l}\text { Mujer, es una de las } \\
\text { pocas cordobezas de la } \\
\text { toma. }\end{array}$ & $\begin{array}{l}\text { TOMA - V03 - } \\
\text { Pueblos Unidos }\end{array}$ & $\begin{array}{l}\text { Pueblos } \\
\text { Unidos }\end{array}$ \\
\hline 4 & $22 / 09 / 2014$ & $\begin{array}{l}\text { Guardería } \\
\text { comunitaria }\end{array}$ & 1 h $36^{\prime}$ & $\begin{array}{l}\text { Hombre, vive en un } \\
\text { barrio aledaño, realiza } \\
\text { trabajo comunitario en } \\
\text { la toma desde } 2009\end{array}$ & $\begin{array}{c}\text { TOMA - } \\
\text { UNIDHOSO1 - } \\
\text { Pueblos Unidos }\end{array}$ & $\begin{array}{l}\text { Pueblos } \\
\text { Unidos }\end{array}$ \\
\hline 5 & $22 / 09 / 2014$ & $\begin{array}{l}\text { Guardería } \\
\text { comunitaria }\end{array}$ & 2 h $12^{\prime}$ & $\begin{array}{c}\text { Mujer, vive en un barrio } \\
\text { aledaño, realiza trabajo } \\
\text { comunitario en la toma } \\
\text { desde } 2009\end{array}$ & $\begin{array}{c}\text { TOMA - } \\
\text { UNIDHOSO2 - } \\
\text { Pueblos Unidos }\end{array}$ & $\begin{array}{l}\text { Pueblos } \\
\text { Unidos }\end{array}$ \\
\hline 6 & 03/10/2014 & En la plaza & $42^{\prime}$ & Mujer, peruana & $\begin{array}{l}\text { TOMA - V04 - } \\
\text { Pueblos Unidos }\end{array}$ & $\begin{array}{l}\text { Pueblos } \\
\text { Unidos }\end{array}$ \\
\hline 7 & 03/10/2014 & $\begin{array}{c}\text { Casa de la } \\
\text { entrevistada }\end{array}$ & $33^{\prime}$ & Mujer, peruana & $\begin{array}{l}\text { TOMA - V05 - } \\
\text { Pueblos Unidos }\end{array}$ & $\begin{array}{l}\text { Pueblos } \\
\text { Unidos }\end{array}$ \\
\hline 8 & $13 / 10 / 2014$ & $\begin{array}{l}\text { Casa de la } \\
\text { entrevistada }\end{array}$ & $1 \mathrm{~h} 2^{\prime}$ & $\begin{array}{l}\text { Mujer, es una de las } \\
\text { primeras habitantes de } \\
\text { la toma. }\end{array}$ & $\begin{array}{l}\text { TOMA - V06 - } \\
\text { Pueblos Unidos }\end{array}$ & $\begin{array}{l}\text { Pueblos } \\
\text { Unidos }\end{array}$ \\
\hline
\end{tabular}




\begin{tabular}{|c|c|c|c|c|c|c|}
\hline Número & Fecha & Lugar & $\begin{array}{l}\text { Duración de } \\
\text { la entrevista }\end{array}$ & Datos & Código & Caso \\
\hline 9 & $16 / 10 / 2014$ & $\begin{array}{l}\text { Caminando por el } \\
\text { barrio }\end{array}$ & $1 \mathrm{~h}$ & $\begin{array}{l}\text { Mujer, es una } \\
\text { referente comunitaria } \\
\text { peruana, cercana a la } \\
\text { organización Un Techo } \\
\text { para mi País. }\end{array}$ & $\begin{array}{l}\text { TOMA - V07 - } \\
\text { Pueblos Unidos }\end{array}$ & $\begin{array}{l}\text { Pueblos } \\
\text { Unidos }\end{array}$ \\
\hline 10 & $18 / 10 / 2014$ & $\begin{array}{c}\text { Casa del } \\
\text { entrevistado }\end{array}$ & $47^{\prime}$ & $\begin{array}{l}\text { Hombre, boliviano, } \\
\text { trabaja de albañil. }\end{array}$ & $\begin{array}{l}\text { TOMA - V08 - } \\
\text { Pueblos Unidos }\end{array}$ & $\begin{array}{l}\text { Pueblos } \\
\text { Unidos }\end{array}$ \\
\hline 11 & $18 / 10 / 2014$ & $\begin{array}{c}\text { Casa del } \\
\text { entrevistado }\end{array}$ & $1 \mathrm{~h} 20^{\prime}$ & $\begin{array}{l}\text { Hombres bolivianos. } \\
\text { Estuvieron desde el } \\
\text { inicio de la toma. } \\
\text { Ignacio es delegado de } \\
\text { manzana. }\end{array}$ & $\begin{array}{l}\text { TOMA - V09 - } \\
\text { Pueblos Unidos }\end{array}$ & $\begin{array}{l}\text { Pueblos } \\
\text { Unidos }\end{array}$ \\
\hline 12 & $03 / 11 / 2014$ & $\begin{array}{l}\text { Casa de uno de los } \\
\text { entrevistados }\end{array}$ & $51^{\prime}$ & $\begin{array}{c}\text { Mujer y Hombre } \\
\text { peruanos. Nuevos } \\
\text { pobladores de la toma. }\end{array}$ & $\begin{array}{l}\text { TOMA - V10 - } \\
\text { Pueblos Unidos }\end{array}$ & $\begin{array}{l}\text { Pueblos } \\
\text { Unidos }\end{array}$ \\
\hline 13 & 03/11/2014 & $\begin{array}{l}\text { Casa de la } \\
\text { entrevistada }\end{array}$ & $44^{\prime}$ & $\begin{array}{c}\text { Mujer peruana, } \\
\text { trabajó en la guardería } \\
\text { comunitaria. }\end{array}$ & $\begin{array}{l}\text { TOMA - V11 - } \\
\text { Pueblos Unidos }\end{array}$ & $\begin{array}{l}\text { Pueblos } \\
\text { Unidos }\end{array}$ \\
\hline 14 & $06 / 11 / 2014$ & Universidad & $1 \mathrm{~h} 2^{\prime}$ & $\begin{array}{l}\text { Mujer, voluntaria de Un } \\
\text { Techo Para Mi País }\end{array}$ & $\begin{array}{l}\text { TOMA - Techo - } \\
\text { Pueblos Unidos }\end{array}$ & $\begin{array}{l}\text { Pueblos } \\
\text { Unidos }\end{array}$ \\
\hline 15 & $17 / 11 / 2014$ & $\begin{array}{l}\text { Casa de la } \\
\text { entrevistada }\end{array}$ & $2 \mathrm{~h}$ & Mujer argentina & $\begin{array}{l}\text { TOMA - V12 - } \\
\text { Pueblos Unidos }\end{array}$ & $\begin{array}{l}\text { Pueblos } \\
\text { Unidos }\end{array}$ \\
\hline 16 & $05 / 02 / 2015$ & $\begin{array}{c}\text { Casa de la } \\
\text { entrevistada }\end{array}$ & $2 \mathrm{~h}$ & $\begin{array}{c}\text { Mujer, organizada en } \\
\text { VUDAS }\end{array}$ & $\begin{array}{c}\text { VUDAS- Vecinas } \\
1\end{array}$ & $\begin{array}{l}\text { VUDAS } \\
\text { contra } \\
\text { Porta. }\end{array}$ \\
\hline 17 & $13 / 03 / 2015$ & $\begin{array}{c}\text { Casa de una de las } \\
\text { entrevistadas }\end{array}$ & $56^{\prime}$ & $\begin{array}{c}\text { Mujeres referentes de } \\
\text { VUDAS }\end{array}$ & $\begin{array}{c}\text { VUDAS- Vecinas } \\
2\end{array}$ & $\begin{array}{l}\text { VUDAS } \\
\text { contra } \\
\text { Porta. }\end{array}$ \\
\hline 18 & $22 / 03 / 2015$ & $\begin{array}{l}\text { Casa de una de las } \\
\text { entrevistadas }\end{array}$ & $58^{\prime}$ & $\begin{array}{l}\text { Mujer organizada en } \\
\text { VUDAS }\end{array}$ & $\begin{array}{c}\text { VUDAS- Vecina } \\
3\end{array}$ & $\begin{array}{l}\text { VUDAS } \\
\text { contra } \\
\text { Porta. }\end{array}$ \\
\hline 19 & $14 / 04 / 2015$ & $\begin{array}{l}\text { Casa de la } \\
\text { entrevistada }\end{array}$ & $1 \mathrm{~h} 15^{\prime}$ & $\begin{array}{c}\text { Mujer organizada en } \\
\text { VUDAS }\end{array}$ & $\begin{array}{c}\text { VUDAS- Vecina } \\
4\end{array}$ & $\begin{array}{l}\text { VUDAS } \\
\text { contra } \\
\text { Porta. }\end{array}$ \\
\hline 20 & $07 / 04 / 2015$ & $\begin{array}{l}\text { Casa de una de las } \\
\text { entrevistadas }\end{array}$ & $41^{\prime}$ & $\begin{array}{c}\text { Mujer organizada en } \\
\text { VUDAS }\end{array}$ & $\begin{array}{l}\text { VUDAS- Vecina } \\
5\end{array}$ & $\begin{array}{l}\text { VUDAS } \\
\text { contra } \\
\text { Porta. }\end{array}$ \\
\hline
\end{tabular}

Fuente: Autora (2018).

El análisis de los datos se ha realizado recuperando herramientas del análisis de contenido. Ello implicó el abordaje de los datos a partir de la emergencia de categorías "en vivo", entrelazándolas y tensionándolas con la literatura que acompaña a nuestro objeto de estudio. Como resultante, obtuvimos una clave de interpretación de esos datos que involucra múltiples discursos: el teórico, el de los actores, y el nuestro.

Diseñamos una estrategia de aproximación consistente en varios momentos. En un primer momento se procedió al análisis de contenido de los datos generados durante el trabajo de campo para luego comenzar a indizar algunos elementos discursivos que pudiesen estar advirtiendo sobre regularidades y relaciones entre los conflictos analizados. En una segunda instancia se abordó cada caso específico a los fines de dar cuenta de sus particularidades y características. Este proceso condujo, muchas veces, a encontrar discursos que excedían, en todo o en parte, los marcos conceptuales, exigiendo un ejercicio analítico consistente en un cuidadoso proceso de producción de categorías de manera tal de fundar empíricamente las interpretaciones que hemos ido elaborando. En otras situaciones esos marcos 
permeaban nuestro abordaje tensionando nuestra interpretación, operando recortes específicos sobre los datos, denunciando ausencias y exigiendo la reapertura del trabajo de campo (AVALLE, 2014).

En otras palabras, desarrollamos un trabajo en dos niveles o en dos momentos: primero el abordaje transversal de los casos para, a partir de ello, construir dimensiones analíticas que nos permitieron la comparación de los casos. Un segundo momento donde cada caso fue abordado de modo específico con el objetivo de identificar sus rasgos característicos. Este ejercicio posibilitó la sistematización de los datos y la comparación entre los casos.

La secuencialidad con que aquí se presenta el método es sólo expositiva, pues este implicó un permanente desplazamiento entre el dato y la conceptualización construida a partir de este. Dicha operatoria fue posible gracias a la utilización de un complejo herramental articulado en torno a nuestros objetivos de análisis.

\section{SIGNIFICACIONES SOBRE LA INSEGURIDAD AMBIENTAL}

Aquí se analizan los discursos de los casos estudiados que aceptan la perspectiva científica y la presencia de polución. Particularmente nos hemos concentrado en la diversa significación sobre la polución que emerge en cada caso analizado. Hemos denominado inseguridad ambiental a la exposición de los cuerpos a ambientes que los ponen en peligro, aquellos espacios que por sus características ponen en "riesgo" la continuidad biológica de los cuerpos.

En el caso de Pueblos Unidos, quienes aceptan la presencia de polución relativizan su importancia en virtud de otras amenazas en la vida cotidiana relacionadas a la precariedad entendida como exposición, falta de protección y escasez de opciones para hacer la vida posible (LOREY, 2016). Mientras que, en el caso de VUDAS la precariedad no se expresa a partir del innacceso a recursos que permitan la vida sino como la propia exposición a los agentes contaminantes.

En el caso de los discursos de Pueblos Unidos que aceptan la existencia de polución, la percepción de actualidad o potencialidad de enfermedad asume diversos sentidos:

EO1: Volaron, pero además empezaron a batallar en contra, digamos, sin, sin plantear ninguna alternativa. ¿Con qué cara te podés parar a la gente y decir, "escuchá, usted no puede vivir acá porque esto está contaminado"? Muy bien. ¿Qué hacemos? ¿Cuál es la alternativa? Y a mí me alucinaba porque yo me acuerdo mamás diciendo: "haber, estas tierras están contaminadas. Suponte que está contaminada"(-) porque nadie les cree además. "Suponte que esté contaminada, pero yo prefiero tener un hijo en estas tierras contaminadas y que nos moriremos de acá a diez, veinte años, a que se me mueran en una semana viviendo abajo del puente." Y era absolutamente lógico el planteo porque si vos no le estás ofreciendo una alternativa de solución, ¿para qué le vas a decir "vos de acá te tenés que ir"? (PUEBLOS UNIDOS - 18:42).

En este extracto la aceptación del discurso científico está atravesada por las condiciones materiales de los cuerpos que allí habitan. La vida se encuentra expuesta a la precariedad por lo cual se produce una escasez en las opciones para garantizarla que se expresa en la reiterada alusión al léxico "alternativa".

En el discurso se construye una dimensión temporal particular. Un "aquí", vinculado al presente, marcado por la inseguridad ambiental donde la enfermedad es percibida como posible pero no segura $y$, por otra parte, un futuro incierto, desconocido, contingente, donde la posibilidad de ser expulsados a territorios menos vivibles aparece como concreta. Allí, la amenaza y la exposición que supondría perder la tierra es percibida como un riesgo mayor para la integridad del cuerpo. La polución de estas tierras plantea una situación de riesgo potencial que se opone a la situación de riesgo de hecho por la que atravesarían si perdieran sus hogares.

Es decir, se configuran dos dimensiones temporales relacionadas a la posibilidad de muerte. Se produce un cálculo estratégico por parte de los sujetos, que debe ser entendido como el intento por retardar la posibilidad de muerte a través de la "selección" de aquellas opciones que permitan una protección inmediata. La vida se encuentra cercada por las amenazas de la exposición: al frío, al agua, a la polución 
y a la enfermedad. La opción estratégica que algunos de los entrevistados proponen se asienta sobre un imaginario del territorio como refugio de la vida, incluso cuando este se encuentre contaminado. Supone la posibilidad de afirmar la vida, aún cuando la muerte por polución al mediano plazo sea una certeza:

No y ella no nos quiere (en referencia a la trabajadora social del UPAS 15). Y de verdad nosotros la hemos combatido bastante, ¿sí? Porque era terrorífico. Yo un día le dije: "¿pero vos sos asistente social o sos del Éter? (se ríen) ¿Viste? “¿De dónde saliste?” "No Alba -dice- pero no pueden vivir ahí". "A ver. Suponte que no se pueda vivir ahí, ¿cuál es tu propuesta? ¿Cuál es tu alternativa?" ¿Sabés que nos dijeron? ¿Qué este plan lo habían hecho la gente de Cáritas? Uno de Cáritas que venía acá a laburar al barrio que era ingeniero y que el hijo tenía una empresa que hace inteligencia sobre tierra y que lo quiso ver y bueno. Estaba en el negocio inmobiliario. No sé qué tiene que ver con esto. ¿Cuál es el negocio suyo de no querer que nosotros estemos acá? Eh. Y entonces viene un día con una nota y una propuesta y le teníamos que firmar de que nos íbamos de acá, y nos íbamos a ir a tomar unas tierras que estaban allá que, donde antes se tomaba, se sembraba soja y eran once, doce hectáreas como este predio pero allá no estaba contaminado. Mirá. Casi me dio un ataque. Encima donde se sembraba soja. Primero que tiene dueño privado. Y ese sí, entramos y al rato cae la guardia de infantería, la gendarmería y nos saca a todos de los pelos. O sea, iQué de los pelos! A puro, a puro balazos digamos. Eh, pero además nos llevaba a un campo de soja que venía de estar fumigado del año pasado. ¿Entendés? A ver ¿Cuál es el chiste? Acá tenemos la basura muerta de treinta, cuarenta años y ponele que haya algún residuo de metales pesados y qué sé yo. Y lo respiramos en el ambiente. ¿Es chiste? ¿Qué quieren inventar? (PUEBLOS UNIDOS - 18:6).

La vida está constreñida por una concatenación de expulsiones/exclusiones que reducen las opciones de los sujetos. Así, la tierra para el negocio pone en juego aquel territorio que las y los vecinos produjeron como refugio.

Se trata del cálculo que procura la continuidad de la vida, su protección aún cuando ello implique "elegir el mal menor". En este discurso, los efectos nocivos de la polución aparecen como relativos en comparación con la ausencia de posibilidades para hacer posible la vida. Así, la precariedad impone un cálculo a los cuerpos, la opción estratégica entre diversas posibilidades de muerte, en donde la selección se guía por la posibilidad de extender la vida, de mantener el cuerpo lo más íntegro posible, generar condiciones de protección de la vida en el mediano plazo y reducir la precariedad. Es por ello que discrepamos con D'hers $(2011$, p. 2) que sostiene:

Es posible vincular la configuración de las sensibilidades sociales en ámbitos contaminados, con cierta política de los cuerpos, y sistemático acostumbramiento a condiciones de habitabilidad precaria. Buscando comprender no sólo por qué es que se forman barrios sobre terrenos no aptos para la vivienda, sino cómo es que esto es posible, y más aún para muchos habitantes de nuestro país deseado en tanto significa no alquilar casas en villas (signadas por la estigmatización social), no vivir en espacios sujetos a condiciones de violencia creciente, y percibir una mejora en su trayectoria habitacional. Comprender que esto es parte de una forma de dominación social resulta vital para cualquier sociedad que se llame a sí misma democrática (D'HERS, 2011, p.2).

Por el contrario, del análisis de las entrevistas de los conflictos aquí estudiados se desprende que permanecer y vivir en territorios "contaminados" no se explica por un "acostumbramiento", sino por un cálculo estratégico que persigue el objetivo de proteger la vida. Coincidimos en que la inseguridad ambiental debe ser entendida como una forma de dominación social. Sin embargo, caracterizar a estos sujetos como "acostumbrados" supone una idea pasiva, que suspende su agencia.

Por el contrario, aquí sostenemos que se trata de sujetos que, encerrados por la falta de opciones, relativizan la potencialidad de polución frente a otras desprotecciones percibidas como más concretas. Lejos de ser sujetos pasivos, acostumbrados, son sujetos imbuidos en la lucha cotidiana por la supervivencia, cuando han sido empujados a ambientes inhóspitos. Se trata de prácticas resistentes cotidianas destinadas a hacer posible la vida.

Así, el cuerpo aparece como el propio agente de protección a partir del cálculo estratégico que aumenta las probabilidades de vida. Es preciso señalar los dispositivos de poder que exponen a los cuerpos, que 
los expulsan a estos territorios como un modo de "dominación social". Al mismo tiempo, es necesario sostener que la "elección" no se encuentra anclada al análisis costo beneficio en términos racionales (ELSTER, 1990; OLSON, 1995) sino, más bien, por el cálculo muerte/vida-protección/desprotección donde la administración de las probabilidades permite alargar la vida.

Así, la lógica de las decisiones se vincula con maximizar la duración de la vida teniendo como criterio el tiempo. Resulta preferible vivir sobre una basural contaminado con residuos que datan de hace 30 años, a vivir en un campo fumigado recientemente o bajo un puente. En ese hiato temporal se incrementa la posibilidad de que la tierra se haya "limpiado" por sí sola, aunque solo es una posibilidad, representa una esperanza. Esta esperanza se expresa en el reiterado uso del condicional simple que supone una hipótesis sujeta a consideraciones. A su vez, la decisión de permanecer en pos de preservar la vida también se encuentra relacionada a evitar nuevas exposiciones frente a la policía. La posibilidad de avanzar sobre otras tierras implica una nueva exposición en la lucha3.

Por otra parte, en los discursos, la polución se presenta como una constante urbana de la cual no es posible escapar:

EO1: Sí, eso que vos decís, te lo digo en una anécdota que hay. Yo como ser, soy con un grupo de diez vecinos más o menos, hemos presentado una denuncia contra Aguas Cordobesas, Schiaretti que era el gobernador, Giacomino y los sojeros por la contaminación del canal a cielo abierto donde 400 mil cordobeses toman agua. Nosotros presentamos y yo le decía a los vecinos "Hemos hecho esa denuncia y a la vez estamos luchando para que nos den agua para Pueblo Unidos". Pero bueno, son como que nos cercan o los límites que nos pone el mismo capitalismo. Estamos peleando para que nos den agua contaminada. Bueno, pero si no nos dan. O sea, eso cualquier, bueno, ya lo empiezan a decir varios de la Universidad, hay 70 kilómetros de ese canal a cielo abierto donde los mosquitos se lavan en ese canal que después la tomamos al agua. Y además que, eh, pasan entre las plantaciones de soja, entonces pasan los aviones, fumigan y está cayendo todo al canal. Y los bidones, ni siquiera tienen ese pudor para hacerlo, los bidones desocupados de agroquímicos lo tiran al canal (PUEBLOS UNIDOS - 17:16).

En estos discursos la polución es característica del capitalismo, un modo de producción que necesariamente intoxica todas las zonas de la ciudad por igual. La vida se encuentra "cercada" por la polución. Los sujetos se encuentran atravesados por la paradoja de "pelear por cosas contaminadas". Los usos del suelo que el capital, en sus diversas fracciones, demanda y produce, encierran a los cuerpos y su posibilidad de desarrollo sano. La producción de soja y el uso de pesticidas, el desarrollo de basurales para el descarte del consumo, la producción de territorios para el negocio y no para la vida colocan a los sujetos en la necesidad constante de supervivencia. En este contexto entonces asume sentido pelear para proteger y extender la vida todo lo posible.

Por su parte, en el conflicto VUDAS la inseguridad ambiental es producida por la actividad de la empresa Porta Hnos. que enfrenta la vida a múltiples amenazas:

Era lo que le decíamos al intendente, no queremos ser otro Barrio Ituzaingó, no queremos esperar hasta que haya 200 casos de cáncer o de muertes para que recién reaccionen y activen. Creemos que con lo que ya tenemos es suficiente para parar el cese de la producción del bioetanol, por que los químicos ya los tenemos todos en la sangre, vamos a ir cayendo a medida que las defensas se vayan bajando, los que tengan las defensas bajas son los primeros, los que estén cerca y hayan respirado, por que al principio empezaron a producir sin filtro ellos, sin nada, entonces esto caía puro al aire. EO2: Estamos hablando de contaminación ¿Y el riesgo de explosión? EO1: No,de eso todavía no hemos hablado. Lo que es el riesgo de explosión si producen 100 mil litros diarios de bioetanol acá, ya han habido incendios en la fabrica y bueno, después de lo que pasó en Raponi estamos todos más alarmados, alterados, ellos que están al lado las escuchan muchos más fuertes, constantemente. Entonces es vivir en estado de miedo, pánico, cuando no sabes si te vas a enfermar, si va a explotar Porta ¿Qué va a pasar viste? es muy estresante vivir con miedo (VUDAS- 52:21).

La inseguridad ambiental, en este extracto, se encuentra relacionada al miedo, al estrés, a la incertidumbre, lo contingente e impredecible que expone la vida a la muerte. La exposición es por la polución y también por la posibilidad de explosión de la fábrica productora de alcohol- Porta Hnos- y la catástrofe que podría arrasar con buena parte de la ciudad. 
Se construye en el discurso la idea de que la enfermedad es una amenaza latente que acecha y es impredecible. Se trata de la imposibilidad de predecirla pero la seguridad de que aparecerá ya que el cuerpo ha sido expuesto y tarde o temprano presentará síntomas. Es un cuerpo suspendido entre la salud y la enfermedad en tránsito por un camino que lo llevará a la muerte por polución.

La temporalidad aquí se erige en otro sentido que aquel del conflicto de Pueblos Unidos. La enfermedad se presenta como un hecho, aquello que ya ha ingresado al cuerpo, lo transita y recorre aunque no se haya manifestado aún. Así, el cuerpo transita un camino entre vida-muerte en donde esta última se va apoderando de él hasta desgastarlo y extinguirlo. Esta progresividad se expresa en el uso del futuro próximo, en articulación con el presente continuo: "van a ir cayendo", "vayan bajando". Si bien esta característica es generalizada en todos los cuerpos, aquí se trata de una particularidad, de una profundización e inducción de aquello que sucede naturalmente fruto del accionar humano. Al mismo tiempo, se trata de un tiempo incierto de espera, controlado por otros (funcionarios, doctores, personal de la compañía), un "tiempo alienado" (AUYERO Y SWISTUN, 2007).

El cuerpo no presenta efectos inmediatos, es un proceso lento y silencioso que lo somete a la desprotección y a defenderse como organismo. Resulta interesante la expresión "los que tengan las defensas bajas son los primeros" en tanto remite al cuerpo que debe contar con condiciones propias que hagan posible la defensa frente a "ataques". Este imaginario puede ser incluso comprendido como analogía a la guerra, el cuerpo que se encuentra compelido a la lucha, al conflicto por la defensa de su vida.

Así mismo, la idea de que los que vivan más cerca de la fábrica serán los primeros en morir remite a un ideario de territorio de sacrificio (SVAMPA, 2008) conformado por niveles de exposición anclados territorialmente. Estos niveles de exposición aparecen en el discurso como el origen de múltiples inseguridades que no solo refieren al ambiente propiamente dicho sino que, además refiere a la posibilidad de explosión. La muerte asume una dimensión territorial al configurar espacios de mayor o menor riesgo de acuerdo a la cercanía/lejanía al origen de la producción de muerte: la fábrica.

En el caso de VUDAS, el "cuerpo enfermo" es una marca que opera sobre la subjetividad pero que asume la forma de la automarca. Es la vivencia interna de la enfermedad que desafía incluso los cánones de medición: allí donde la ciencia vinculada a los intereses del capital sostiene que no hay rastros de enfermedad, los cuerpos sostienen su presencia. En sus cuerpos se expresan los resultados de las dinámicas productivas, de los procesos extractivos y de las lógicas de explotación y contaminación de los recursos (SOLANO, 2014). Así, frente al riesgo de multiplicación de la enfermedad solo queda lo colectivo y la unión como condición de victoria.

En síntesis, el principal articulador de los relatos del conflicto VUDAS- Porta hnos. es el léxico "hay". Se trata de la constante alusión a "hay polución, enfermedad, químicos", se relaciona con la presencia de elementos que "atacan" la vida. Es la vida puesta en juego en su capacidad y posibilidad de supervivencia: "hay muerte". Por el contrario, el caso de Pueblos Unidos se relaciona con el léxico "no hay", como falta o desprotección por abandono, la referencia a la escasez que se expresa en inaccesos a opciones y recursos por haber sido excluido del mercado.

\section{REFLEXIONES FINALES}

Los modos en los cuales las comunidades se relacionan con la polución se encuentra mediado por procesos sociales de producción de sentido y significación. No existen comunidades a priori dispuestas a luchar contra la polución, ni comunidades que hayan naturalizado por completo las condiciones del ambiente en el que viven. Así, es preciso analizar cada caso a los fines de conocer qué elementos, contextos y narrativas funcionan como marco que permite dotar de sentido lo que es considerado como problemático.

En ambos casos analizados la inseguridad ambiental se significa de modo diverso. Esta diversidad se explica por la singular expresión de precariedad que se construye en cada caso.

En el conflicto de Pueblos Unidos, la inseguridad ambiental en la que se encuentran los cuerpos contrasta con la a precariedad que encorseta la vida. Las opciones para la supervivencia son escasas 
por lo cual emerge un cálculo estratégico que se basa en la noción de temporalidad y posibilidad. Así, la polución es aceptada por una parte de la comunidad barrial y sus efectos sobre el cuerpo aparecen como potenciales en comparación con la posibilidad de muerte que supone la renuncia a este lugar y encontrarse a la intemperie, sin nada, "debajo de un puente".

La vida precaria sopesa opciones que le permitan prolongar el tiempo vital, allí donde las condiciones de vida son adversas y la exposición es total. La exposición total remite a la imbricación que se produce entre diversas dimensiones de la vida -laboral, habitacional. El tener trabajo intermitente e informal dificulta la posibilidad de contar con una casa, lo cual redunda en historias de vida marcadas por la exclusión.

Por su parte, en el conflicto VUDAS- Porta hnos. la polución aparece como una evidencia que enferma cotidianamente los cuerpos. La precariedad en este caso no se relaciona con la ausencia de opciones o con la escasez, sino, propiamente, con la polución. La temporalidad de la enfermedad aparece como actual, aunque silenciosa. Se trata del cuerpo que se encuentra enfermo aún cuando no presente síntomas de ello. El cuerpo asume la marca de la enfermedad, la reivindica y la constituye en denuncia. Este cuerpo enfermo es aquel que ha sido atacado por la polución y que no tiene escapatoria posible. La única posibilidad es la lucha por la defensa de la vida de quienes aún no han sido enfermados.

La lucha, entonces, asume un doble sentido, por la erradicación de la empresa, para evitar que la amenaza se extienda a cada vez mayores fracciones de la población, pero al mismo tiempo, una lucha orgánica e interna por fortalecer el propio cuerpo y el de los hijos, instar al organismo a contar con las defensas necesarias para la supervivencia. El cuerpo es sometido a aquello que resulta insoportable y que progresivamente va matándolo.

En conclusión, cada comunidad significa la inseguridad ambiental de modo diverso. Esta diversidad se relaciona con las historias de inclusión/exclusión de las poblaciones estudiadas. De allí que la precariedad emerja como una característica de los casos estudiados pero asume dinámicas singulares. La particular expresión de precariedad de cada caso es lo que da lugar a las significaciones que emergen en los discursos analizados.

\section{NOTAS}

${ }^{1}$ Este caso representa uno de los mayores antecedentes en la lucha medio ambiental en Argentina. Este barrio es el más extenso de la periferia de la ciudad y colinda con campos de cultivo. Por esta razón, las fumigaciones eran constantes y han ocasionado muertes, malformaciones en bebés e intoxicación en la población. A partir de las movilizaciones de las Madres, se declaró la emergencia sanitaria del barrio y la promulgación de ordenanzas tanto para el barrio como para toda la provincia que crean franjas de protección contra las fumigaciones. A su vez, las madres organizadas lograron que en el año 2012 se realizara un juicio por fumigaciones ilegales, donde fueron declarados culpables un productor sojero y un aeroaplicador.

${ }^{2}$ Los códigos que se encuentran al final de los extractos de entrevista son identificatorios. El primer número refiere a la entrevista mientras que el segundo da cuentas del extracto. Ejemplo: (7:1) entrevista 7, cita 1.

${ }^{3}$ La mayoría de las experiencias de tomas de tierra en la Provincia de Córdoba han sido profundamente reprimidas y judicializadas por el Estado, registrando altos niveles de violencia y ocupación territorial por parte de las fuerzas policiales mientras duró el conflicto. Al respecto revisar Tomas de tierras en Córdoba. Informe Preliminar (2013). Colectivo de investigación El Llano en Llamas.

\section{BIBLIOGRAFÍA}

ÁlVAREZ-GAYOU, J. L. Cómo hacer investigación cualitativa. Fundamentos y metodología. D.F.: Paidós Mexicana, 2003.

AUYERO, J. y SWISTUN, D. Inflamable: Estudio del sufrimiento ambiental. Buenos Aires: Paidós, 2008. 
AUYERO, J. y SWISTUN, D. Expuestos y confundidos. Un relato etnográfico sobre sufrimiento ambiental. Iconos, Revista de Ciencias Sociales, Quito, n. 28, p. 137-152, 2007.

AVALLE, G. La contienda de la educación: lucha y acción colectiva sindical en la Argentina contemporánea, análisis de tres contiendas provinciales de sindicalismo docente. Córdoba: EDUCC, 2014.

BECK, U. La sociedad del riesgo: hacia una nueva modernidad. Barcelona: Paidós, 1998.

BERGER, M. Cuerpo, experiencia, narración. Auto-organización ciudadana en situaciones de contaminación ambiental. Córdoba: Ed. del Boulevard, 2013.

BUTTEL, F. New directions in environmental sociology. Annual review of sociology, Stanford, v. 13 n. 1, p. 465-488, 1987.

CBA 24N "Acusan que el 50\% del barrio está enfermo por planta de Porta". 13 de Agosto de 2013. Dispoible en: http://www.cba24n.com.ar/content/acusan-que-el-50-del-barrio-esta-enfermo-porplanta-de-porta. Recuperado: 27 de abril de 2017.

COLECTIVO DE INVESTIGACIÓN EL LLANO EN LLAMAS, Informe preliminar, Tomas de tierras en Córdoba. (2013)

Disponible en: http://www.llanocordoba.com.ar/publicaciones/informes/345-toma-de-tierras-encordoba-informe-preliminar-mayo-2013-54949872. Recuperado: 15 de Marzo 2017.

D'HERS, V. Configuraciones de las sensibilidades y soportabilidad social en hábitats precarios. Lomas de Zamora, provincia de Buenos Aires (2007-2011). Tesis Doctoral. Buenos Aires: Universidad de Buenos Aires, 2011.

DOUGLAS M. y AARON W. Risk and Culture. An Essay on the Selection of Technological and Environmental Dangers. Los Ángeles: University of California Press, 1982.

DUNLAP, R. y MICHELSON, W. Handbook of environmental sociology. Westport: Greenwood Publishing Group, 2002.

ECOS CÓRDOBA. "Presentan una medida judicial “autosafisfactiva”. 23 de Diciembre de 2013. Disponible en: http://ecoscordoba.com.ar/presentan-una-medida-judicial-autosafisfactiva/. Recuperado: 10 de Marzo de 2017.

ECOS CÓRDOBA “PORTA Hnos. S A: En la mira de la Responsabilidad Social”. 20 de marzo de 2015. Disponible en: http://ecoscordoba.com.ar/porta-hnos-s-a-en-la-mira-de-la-responsabilidad-social/. Recuperado 10 de Diciembre de 2016.

ELSTER, J. Racionalidad, moralidad y acción colectiva, Zona Abierta, Madrid, v. 54 n. 55, p. 43-67, 1990.

EVANS, P. Livable Cities? Urban Struggles for Livelihood and Sustainability. California: University of California Press, 2002.

GIDDENS, A. The Consequences of Modernity. Stanford: Stanford University Press, 1990.

HOCHSTETLER, K. y KECK, M. The Greening of Brazil. Environmental Activism in State and Society. Durham: Duke University Press, 2007.

INGLEHART, R. The silent revolution in Europe: Intergenerational change in post-industrial societies. American political science review, Nueva York, v. 65 n. 4, p. 991-1017, 1971.

LA TINTA. A un mes de la audiencia, vecinos exigen respuesta judicial al caso Porta. 8 de Septiembre de 2017.

Disponible en: https://latinta.com.ar/2017/09/audiencia-vecinos-exigen-respuesta-porta. Recuperado: 10 de Octubre 2017. 
LA VOZ DEL INTERIOR. Aún hay 200 industrias fuera de norma. 08 de Noviembre de 2014. Disponible en: http://www.lavoz.com.ar/ciudadanos/aun-hay-200-industrias-fuera-de-norma. Recuperado: 12 de Noviembre de 2016.

LA VOZ DEL INTERIOR. Siguen de cerca las enfermedades. 6 de Septiembre de 2009. Disponible en: http:// archivo.lavoz.com.ar/09/09/06/secciones/grancordoba/nota.asp?nota_id=548474. Recuperado: 21 de Noviembre de 2017.

LEMOS, M. C. The Politics of Pollution Control in Brazil: State Actors and Social Movements Cleaning Up Cubatao. World Development, Nueva York, v. 26 n. 1, p. 75- 87, 1998.

LEZAMA, J. L. El medio ambiente como construcción social: reflexiones sobre la contaminación del aire en la Ciudad de México. Estudios Sociológicos, D. F. v. 19 n. 56, p. 325-338, 2001.

LOREY, I. Estado de Inseguridad: Gobernar la precariedad. Madrid: Traficantes de Sueños, 2016.

MACHADO IBARS, M. Allí, y a pesar de la contaminación, la vida ha de enmarcarse como posible. In CIUFFOLINI, M. A. (comp.). Lucha por la tierra. Contexto e historias de las tomas en Córdoba. Córdoba: EDUCC, 2017. p. 81-89.

MUÑIZ, M. Estudios de caso en la investigación cualitativa. División de Estudios de Posgrado Universidad Autónoma de Nuevo León. México, 1-8, 2010.

NEIMAN G, QUARANTA G. Los estudios de casos en la investigación sociológica. In: VASILACHIS DE GIARDINO, I. (ed). Estrategias de investigación cualitativa. Barcelona: Gedisa Editorial, 2006. p. 212237.

OLSON, M. La lógica de la acción colectiva. D.F.: Limusa, 1995.

OLTRA ALGADO, C. Sociedad y medio ambiente. Ciudadanos y científicos ante la reforma medioambiental de la sociedad. Barcelona: Universitat de Barcelona, 2006.

PEZZOLI, K. Human Settlements and Planning for Ecological Sustainability: The Case of Mexico City. Cambridge: The MIT Press, 2000.

SOLANO, S. M. Agroquímicos y sufrimiento ambiental: reflexiones desde las ciencias sociales. Revista Reflexiones, San José de Costa Rica, v. 93 n. 1, p. 199-206, 2014.

SVAMPA, M. Cambio de época, movimientos sociales y poder político. Buenos Aires: CLACSO, 2008.

VASILACHIS DE GIARDINO, I. La representación discursiva de los conflictos sociales en la prensa escrita. Estudios sociológicos, p. 95-137, 2005. 